\title{
Closed-Form Breakdown Voltage Model for PD SOI NMOS Devices Considering Impact Ionization of Both Parasitic BJT and Surface MOS Channel Simultaneously
}

\author{
Shih-Chia Lin and James B. Kuo, Fellow, IEEE
}

\begin{abstract}
This paper reports a compact breakdown voltage model for partially depleted (PD) silicon-on-insulator (SOI) n-metal-oxide-semiconductor (NMOS) devices considering BJT/MOS impact ionization. Via the improved current conduction model considering BJT/MOS impact ionization this compact model provides an accurate prediction of the breakdown behavior of the PD SOI NMOS devices as verified by the experimental data and the MEDICI results. Based on the analytical model, when the gate voltage is lowered, the breakdown voltage decreases due to a stronger function of the parasitic BJT. In the subthreshold region, the breakdown voltage increases at a decreased gate voltage due to a weaker function of the parasitic BJT.
\end{abstract}

Index Terms-Complementary metal-oxide-semiconductor (CMOS), compact model, partially depleted (PD), PD breakdown voltage, silicon-on-insulator (SOI), SPICE.

\section{INTRODUCTION}

$\mathbf{F}$ OR a silicon-on-insulator (SOI) metal-oxide-semiconductor (MOS) device with a small channel length, the breakdown behavior due to a high electric field is important [1], [2]. As for a bulk NMOS device, the breakdown behavior of a partially depleted (PD) SOI NMOS device is caused by impact ionization [3], [4]. Different from a bulk NMOS device, impact ionization of both the surface MOS channel and the parasitic BJT is important in determining the breakdown behavior of a PD SOI MOS device. Breakdown behavior of PD SOI complementary metal-oxide-semiconductor (CMOS) devices has been reported [5]-[8]. Analytical breakdown voltage models of PD SOI NMOS devices have been reported before [9], [10]. However, iterations are needed for a solution [9], [10] or floating-body-dependent base-emitter voltage model is not considered [9]. Until now, no closed-form analytical breakdown model could be found. A closed-form analytical breakdown voltage model is helpful in providing a better understanding of the relative importance of the various parameters while engineering a PD SOI CMOS device. In this paper, using the approach for modeling the kink effects [11], [12], a compact breakdown voltage model considering the BJT/MOS impact

Manuscript received April 3, 2002; revised July 10, 2002. This work was supported by grants from the NSERC Research Programs (Canada). The review of this paper was arranged by Editor P. Yang.

The authors are with the Department of Electrical Computer Engineering (DC2737), University of Waterloo, Waterloo, ON N2L 3G1, Canada (e-mail: jbkuo@uwaterloo.ca).

Digital Object Identifier 10.1109/TED.2002.804728

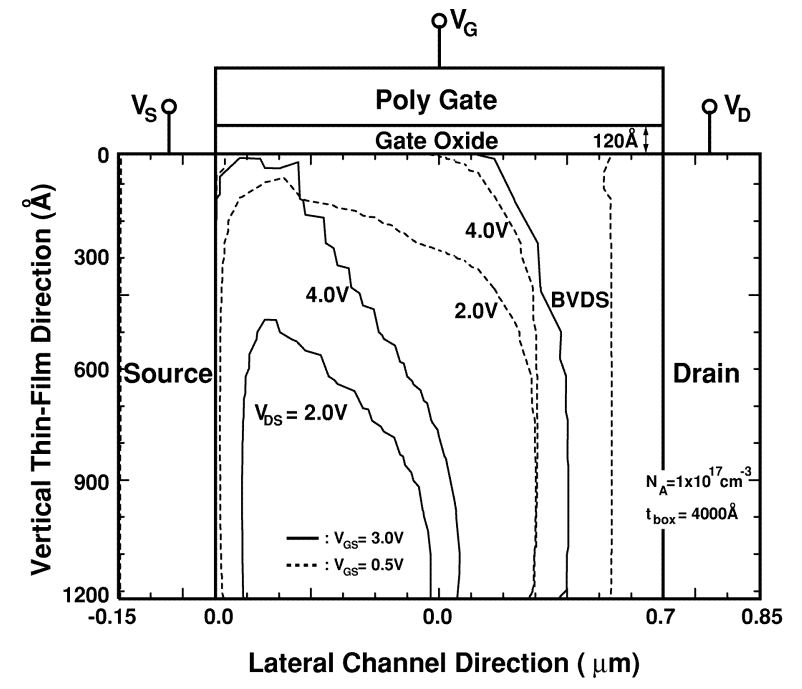

Fig. 1. Two-dimensional contours of hole density of $10^{16} \mathrm{~cm}^{-3}$ in the PD SOI NMOS device with a gate oxide of $120 \AA$, a thin film of $1200 \AA$ doped with a p-type density of $10^{17} \mathrm{~cm}^{-3}$, and a channel length of $0.7 \mu \mathrm{m}$, biased at $V_{\mathrm{GS}}=3 \mathrm{~V}, 0.5 \mathrm{~V}$, and various $V_{\mathrm{DS}}^{\prime} \mathrm{s}$.

ionization for the PD SOI NMOS devices is described. In the following sections, the model derivation is presented first, followed by evaluation of the model, discussion, and conclusion.

\section{BREAKdown Voltage Model}

Fig. 1 shows the two-dimensional (2-D) contours of hole density of $10^{16} \mathrm{~cm}^{-3}$ in a PD SOI NMOS device with a channel length of $0.7 \mu \mathrm{m}$, a gate oxide thickness of $120 \AA$, and a thin film of $1200 \AA$, with a p-type density of $10^{17} \mathrm{~cm}^{-3}$, biased at $V_{\mathrm{GS}}=3 \mathrm{~V}, 0.5 \mathrm{~V}$, and various $V_{\mathrm{DS}}^{\prime} \mathrm{s}$. As shown in Fig. 1 , for the device biased in the strong inversion region at $V_{\mathrm{GS}}=3 \mathrm{~V}$ (solid lines), when $V_{\mathrm{DS}}$ increases from $2 \mathrm{~V}$ to $4 \mathrm{~V}$, the contour of the hole density of $10^{16} \mathrm{~cm}^{-3}$ moves upward, indicating widening of the parasitic BJT. At the breakdown voltage of $V_{\mathrm{DS}}=5 \mathrm{~V}$, from the $10^{16} \mathrm{~cm}^{-3}$ hole density contour, the parasitic BJT occupies most of the thin film. For the device biased in the subthreshold region at $V_{\mathrm{GS}}=0.5 \mathrm{~V}$ (dashed lines), from the $10^{16} \mathrm{~cm}^{-3}$ hole density contours, the function of the parasitic BJT is even stronger as compared to the strong inversion counterpart. Therefore, for deriving the breakdown voltage model, impact ionization of both the surface MOS channel and the parasitic BJT should be included. 


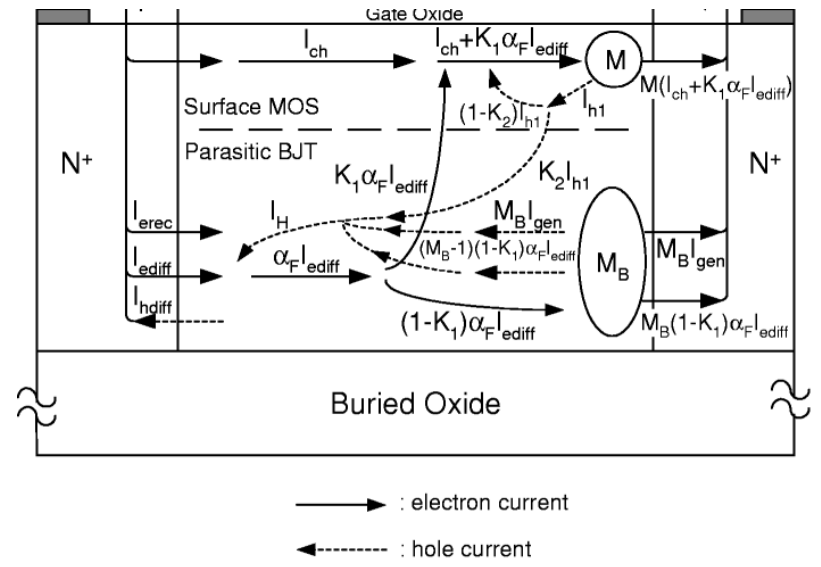

Fig. 2. Current conduction mechanism in a PD SOI NMOS device for deriving the breakdown voltage model.

\section{A. Current Conduction Mechanism}

Fig. 2 shows the current conduction mechanism in a PD SOI NMOS device for deriving the breakdown voltage model. As shown in this figure, the drain current $\left(I_{D}\right)$ is composed of the surface MOS portion (first term) and the parasitic BJT portion (second term) as indicated in the following:

$I_{D}=M\left(I_{\mathrm{ch}}+K_{1} \alpha_{f} I_{\text {ediff }}\right)+M_{B}\left(I_{\text {gen }}+\left(1-K_{1}\right) \alpha_{f} I_{\text {ediff }}\right)$.

For the surface MOS portion, it is the sum of the traditional surface channel current $\left(I_{\mathrm{ch}}\right)$ and a portion $\left(K_{1}\right)$ of the electron diffusion current $\left(I_{\text {ediff }}\right)$ in the neutral base of the parasitic BJT, flowing upward to merge with the channel current, amplified by the multiplication factor $(M)$ due to impact ionization in the high electric field region near the drain- $M\left(I_{\mathrm{ch}}+\right.$ $K_{1} \alpha_{f} I_{\text {ediff }}$ [11], where $K_{1}$ is an empirical parameter [11]. For the parasitic BJT portion, it is the sum of the generation current in the base-collector region $\left(I_{\text {gen }}\right)$ and the remaining portion of the electron diffusion current in the neutral base (1 $\left.K_{1}\right) \alpha_{f} I_{\text {ediff }}$, multiplied by the multiplication factor $\left(M_{B}\right)$ accounting for impact ionization in the high electric field region of the parasitic BJT. In the following subsections, key components in the surface MOS and parasitic BJT portions are described.

\section{B. Surface MOS Channel Current ( $\left.I_{\mathrm{ch}}\right)$}

In the surface MOS channel, depending on the operation regime determined by the gate voltage, its conduction current $\left(I_{\mathrm{ch}}\right)$ varies. When the gate voltage is smaller than the threshold voltage, diffusion current dominates in the surface MOS channel. Therefore, $I_{\mathrm{ch}}$ is made of the diffusion current [13]

$$
\begin{aligned}
I_{\mathrm{diff}} & =\frac{W}{L} I_{x} \exp \left(\frac{V_{G}-V_{x}}{n\left(\frac{k T}{q}\right)}\right)\left[1-\exp \left(-\frac{V_{\mathrm{DS}}}{\left(\frac{k T}{q}\right)}\right)\right] \\
I_{x} & =\mu_{0} C_{\mathrm{ox}}\left(\frac{k T}{q}\right)^{2} \frac{\gamma}{2 \sqrt{1.5 \phi_{f}-V_{\mathrm{BE}}}} \exp \left(-\frac{\phi_{f}}{2\left(\frac{k T}{q}\right)}\right) \\
V_{x} & =V_{\mathrm{FB}}+1.5 \phi_{f}+\gamma \sqrt{1.5 \phi_{f}-V_{\mathrm{BE}}} \\
n & =1+\frac{\gamma}{2 \sqrt{1.5 \phi_{f}-V_{\mathrm{BE}}}} \\
\gamma & =\frac{\sqrt{2 q \epsilon_{\mathrm{si}} N_{A}}}{C_{\mathrm{ox}}}
\end{aligned}
$$

where

$W \quad$ channel width;

$V_{\text {FB }}$ flat-band voltage;

$C_{\mathrm{ox}} \quad$ gate oxide capacitance $\left(C_{\mathrm{ox}}=\epsilon_{\mathrm{ox}} / t_{\mathrm{ox}}\right)$;

$\phi_{f} \quad$ Fermi potential- $\phi_{f}=(k T / q) \ln \left(N_{A} / n_{i}\right)$ for p-type silicon and $\phi_{f}=(k T / q) \ln \left(n_{i} / N_{D}\right)$ for n-type silicon;

$n_{i} \quad$ intrinsic concentration.

When the gate voltage is larger than the threshold voltage, $I_{\mathrm{ch}}$ is dominated by the drift current [11]

$$
\begin{aligned}
I_{\mathrm{drift}}= & \frac{W}{L} \frac{\mu_{0} C_{\mathrm{ox}}}{1+\theta\left(V_{G}-V_{T}\right)-\theta_{B} V_{\mathrm{BE}}} \\
& \times\left[\left(V_{G}-V_{T}\right) V_{\mathrm{dm}}-\frac{1}{2}(1+\delta) V_{\mathrm{dm}}^{2}\right] \\
V_{T}= & V_{\mathrm{FB}}+2 \phi_{f}+\gamma \sqrt{2 \phi_{f}-V_{\mathrm{BE}}} \\
V_{\mathrm{dm}}= & V_{\mathrm{DS}}+V_{\mathrm{DSAT}}-\left(V_{\mathrm{DS}}^{m}+V_{\mathrm{DSAT}}^{m}\right)^{1 / m} \\
V_{\mathrm{DSAT}}= & \frac{E_{C} L\left(V_{G}-V_{T}\right)}{E_{C} L(1+\delta)+\left(V_{G}-V_{T}\right)} \\
\delta= & \frac{\gamma}{2 \sqrt{\phi_{a}+2 \phi_{f}-V_{\mathrm{BE}}}}
\end{aligned}
$$

where $V_{\mathrm{dm}}$ is used to accommodate this general drift current formula for both triode $\left(V_{\mathrm{DS}}<V_{\mathrm{DSAT}}\right)$ and saturation $\left(V_{\mathrm{DS}} \geq\right.$ $\left.V_{\mathrm{DSAT}}\right)$ regions with $m=6$ commonly used. $\mu_{0}, \theta$, and $\theta_{B}$, which are used to describe the mobility behavior, are empirical parameters [13]. $\phi_{a}$ is used to compensate for the deviation caused by Taylor's approximation. $E_{c}$ is the critical electric field.

\section{Parasitic BJT Currents}

In addition to the currents from the surface MOS channel, the currents from the parasitic BJT are also important. As indicated in (1), in the parasitic BJT, the dominant currents are the generation current in the reverse biased base-collector junction $\left(I_{\text {gen }}\right)$ and the electron diffusion current $\left(I_{\text {ediff }}\right)$, which are amplified by the multiplication factor $\left(M_{B}\right)$ of the impact ionization due to the high electric field region in the reverse-biased base-collector junction.

The generation current in the reverse-biased base-collector junction is expressed as

$$
I_{\text {gen }}=\frac{q W_{\mathrm{BC}} n_{i} A_{J}}{2 \tau_{g}}
$$

where $A_{J}$ is the junction area, and $\tau_{g}$ is the generation lifetime in the base-collector junction. $W_{\mathrm{BC}}$ is the width of the depletion region between the floating-body and the drain $\left(W_{\mathrm{BC}}=\sqrt{2 \epsilon_{\mathrm{si}}\left(V_{\mathrm{DS}}+V_{\mathrm{bi}}-V_{\mathrm{BE}}\right) /\left(q N_{A}\right)}\right)$, where $V_{\mathrm{bi}}$ is the built-in voltage of the junction between the source and the thin film $\left(V_{\mathrm{bi}}=(k T / q) \ln \left(N_{A} N_{D} / n_{i}^{2}\right)\right)$, and $V_{\mathrm{BE}}$ is the floating-body potential.

The electron diffusion current in the base of the parasitic BJT is expressed as

$$
\begin{aligned}
I_{\text {ediff }} & =K_{b} \exp \left(\frac{V_{\mathrm{BE}}}{\left(\frac{k T}{q}\right)}\right) \\
K_{b} & =\frac{q D_{\mathrm{eB}} n_{i}^{2} A_{J}}{N_{A} W_{B}}
\end{aligned}
$$


where

$D_{\mathrm{eB}} \quad$ electron diffusion constant $\left(D_{\mathrm{eB}}=(k T / q) \mu_{\mathrm{eff}}\right)$;

$\mu_{\text {eff }} \quad$ electron mobility;

$W_{B}$ effective base width excluding the depletion region of the base-emitter and the base-collector junction $\left(W_{B}=L-W_{\mathrm{EB}}-W_{\mathrm{BC}}\right)$;

$L \quad$ channel length of the SOI device;

$W_{\mathrm{EB}}$ depletion width of the base-emitter junction $\left(W_{\mathrm{EB}}=\right.$ $\left.\sqrt{2 \epsilon_{\mathrm{Si}}\left(V_{\mathrm{bi}}-V_{\mathrm{BE}}\right) / q N_{A}}\right)$.

As indicated in (1), $\alpha_{f}$ is the forward common-base current gain $\left(\alpha_{f}=\alpha_{t} \gamma\right)$ of the parasitic BJT considering the transport factor $\left(\alpha_{t}=1 / \cosh \left(W_{B} / L_{\mathrm{eB}}\right)\right)$ and the injection efficiency [14]

$$
\gamma=\frac{1}{\left(1+\frac{\left(N_{A} D_{\mathrm{pE}} W_{B}\right)}{\left(N_{D} D_{\mathrm{eB}} W_{E}\right)}+\frac{1}{2} \sqrt{\frac{\left(q N_{A} W_{B} A_{J}\right)}{\left(D_{\mathrm{eB}} I_{\text {ediff }}\right)}} \frac{W_{\mathrm{EB}}}{\tau_{r}}\right)} .
$$

$L_{\mathrm{eB}}$ is the electron diffusion length in the base region, which is expressed as $L_{\mathrm{eB}}=\sqrt{D_{\mathrm{eB}} \tau_{\mathrm{eB}}}$, where $\tau_{\mathrm{eB}}$ is the electron lifetime in the base $\left(\tau_{\mathrm{eB}}=\tau_{0} /\left(1+N_{A} / 5 \times 10^{16}\right)\right)$ and $\tau_{0}$ is the hole lifetime in the base.

\section{Impact Ionization}

In the high electric field region after pinchoff in the surface MOS channel, due to impact ionization, the multiplication factor is expressed as

$$
M=1+\frac{\alpha}{\beta}\left(V_{\mathrm{DS}}-V_{\mathrm{DSAT}}\right) \exp \left[-\frac{\beta l}{\left(V_{\mathrm{DS}}-V_{\mathrm{DSAT}}\right)}\right]
$$

where $\alpha, \beta$, and $l$ are impact-ionization-related parameters. Equation (6) is valid for the device in the strong inversion region $\left(V_{G}>V_{T}\right)$. While in the weak inversion region $\left(V_{G}<V_{T}\right)$, $V_{\mathrm{DSAT}}$ should be set to zero. Usually, in the triode region ( $\left.V_{\mathrm{DS}}<V_{\mathrm{DSAT}}\right)$, impact ionization is negligible, thus $M=1$.

In the high electric field region of the base-collector junction in the parasitic BJT, the multiplication factor due to impact ionization is

$$
M_{B}=1+\frac{\alpha_{b}}{\beta_{b}}\left(V_{\mathrm{DS}}-V_{\mathrm{BE}}\right) \exp \left[-\frac{\beta_{b} l_{b}}{\left(V_{\mathrm{DS}}-V_{\mathrm{BE}}\right)}\right]
$$

where $\alpha_{b}, \beta_{b}$, and $l_{b}$ are the impact ionization coefficients.

Due to impact ionization, pairs of electrons and holes are generated in the high electric field region of the surface MOS channel and the parasitic BJT. Due to the positive drain voltage, the negatively charged electrons generated by impact ionization move toward drain to become the drain current $I_{D}$. The generated holes move in the negative direction toward the source terminal, accumulating in the floating-body to cause a floating-body potential. The generated hole current from the impact ionization in the surface MOS region is

$$
I_{h 1}=(M-1)\left(I_{\mathrm{ch}}+K_{1} \alpha_{f} I_{\text {ediff }}\right) .
$$

As shown by the dashed lines in Fig. 2, a portion of the hole current $\left(\left(1-K_{2}\right) I_{h 1}\right)$, generated from the impact ionization in the surface MOS channel, is redirected toward the surface channel. The remaining portion of the generated hole current $\left(K_{2} I_{h 1}\right)$ still flows in the neutral thin film region identified as the floating-body, where $K_{2}$ is a fitting parameter [3]. At the bottom of the thin film, from the high electric field region of the parasitic BJT, impact ionization results in the magnification of the base-collector generation current $\left(I_{\text {gen }}\right)$ and the base electron diffusion current $\left(I_{\text {ediff }}\right)$ amplified by the multiplication factor to become another hole current

$$
I_{h 2}=M_{B} I_{\text {gen }}+\left(M_{B}-1\right)\left(1-K_{1}\right) \alpha_{f} I_{\text {ediff }} .
$$

The total hole current flowing toward the neutral region of the thin film is $\left(I_{H}=K_{2} I_{h 1}+I_{h 2}\right)$

$$
\begin{aligned}
I_{H}=K_{2}(M-1)\left(I_{\mathrm{ch}}+\right. & \left.K_{1} \alpha_{f} I_{\text {ediff }}\right)+M_{B} I_{\text {gen }} \\
& +\left(M_{B}-1\right)\left(1-K_{1}\right) \alpha_{f} I_{\text {ediff }} .
\end{aligned}
$$

\section{E. Breakdown Condition}

In the parasitic bipolar device, the emitter current is composed of the recombination current in the base-emitter junction ( $I_{\text {erec }}$ ), the electron diffusion current $\left(I_{\text {ediff }}\right)$, and the hole diffusion current $\left(I_{\text {hdiff }}\right)$ over the base-emitter junction. The recombination current in the base-emitter junction $\left(I_{\text {erec }}\right)$ is expressed as a function of the base-emitter voltage: $I_{\text {erec }}=K_{a} \exp \left(V_{\mathrm{BE}} / 2(k T / q)\right)$, where $K_{a}=\left(q W_{\mathrm{EB}} n_{i} A_{J}\right) /\left(2 \tau_{r}\right)$, and $\tau_{r}$ is the recombination lifetime in the base-emitter junction. The hole diffusion current over the base-emitter junction $\left(I_{\text {hdiff }}\right)$ is also a function of the base-emitter voltage: $I_{\text {hdiff }}=K_{c} \exp \left(V_{\mathrm{BE}} /(k T / q)\right)$, where $K_{c}=\left(q D_{\mathrm{pE}} n_{i}^{2} A_{J}\right) /\left(N_{D} W_{E}\right), D_{\mathrm{pE}}$ is the hole diffusion constant $\left(D_{\mathrm{pE}}=(k T / q) \mu_{\text {peff }}, \mu_{\text {peff }}\right.$ is the hole mobility $)$, $N_{D}$ is the doping density of the source region, and $W_{E}$ is the emitter width. Note from the above derivation the currents in the parasitic BJT are determined by the base-emitter voltage [11], [12], which is the floating-body potential. The base current is formed by the total hole current $\left(I_{H}\right)$. The collector current is expressed as $\alpha_{f} I_{\text {ediff. In the parasitic bipolar device, }}$ the emitter current is equal to the sum of the collector current and the base current $\left(I_{E}=I_{C}+I_{B}\right)$. Therefore

$$
I_{\text {erec }}+I_{\text {ediff }}+I_{\text {hdiff }}=I_{H}+\alpha_{f} I_{\text {ediff }} .
$$

Since the emitter current is dominated by the electron diffusion current $\left(I_{\text {ediff }}\right)$, (11) can be simplified as $I_{H} \cong\left(1-\alpha_{f}\right) I_{\text {ediff. }}$. Using (10), the electron diffusion current can be expressed as

$$
\begin{aligned}
& I_{\text {ediff }}= \\
& \frac{K_{2}(M-1) I_{\mathrm{ch}}+M_{B} I_{\text {gen }}}{1-\alpha_{f}-K_{1} K_{2}(M-1) \alpha_{f}-\left(M_{B}-1\right)\left(1-K_{1}\right) \alpha_{f}} .
\end{aligned}
$$

From (1) and (12), the drain current becomes

$$
\begin{aligned}
I_{D}= & \frac{f_{1} I_{\mathrm{ch}}+f_{2} I_{\text {gen }}}{f_{0}} \\
f_{0}= & 1-\alpha_{f}-K_{1} K_{2}(M-1) \alpha_{f}-\left(1-K_{1}\right)\left(M_{B}-1\right) \alpha_{f} \\
f_{1}= & M\left(1-\alpha_{f}\right)+\left(1-K_{1}\right) \alpha_{f} \\
& \times\left[K_{2} M_{B}(M-1)-M\left(M_{B}-1\right)\right] \\
f_{2}= & M_{B}\left[1+(M-1) K_{1}\left(1-K_{2}\right) \alpha_{f}\right]
\end{aligned}
$$

Taking a derivative of (13), one obtains

$$
\begin{aligned}
\frac{\partial I_{D}}{\partial V_{\mathrm{DS}}}= & \frac{1}{f_{0}^{2}}\left\{\left(f_{0} \frac{\partial f_{1}}{\partial V_{\mathrm{DS}}}-f_{1} \frac{\partial f_{0}}{\partial V_{\mathrm{DS}}}\right) I_{\mathrm{ch}}\right. \\
& +\left(f_{0} \frac{\partial f_{2}}{\partial V_{\mathrm{DS}}}+f_{2} \frac{\partial f_{0}}{\partial V_{\mathrm{DS}}}\right) I_{\mathrm{gen}} \\
& \left.+f_{0}\left(f_{1} \frac{\partial I_{\mathrm{ch}}}{\partial V_{\mathrm{DS}}}+f_{2} \frac{\partial I_{\mathrm{gen}}}{\partial V_{\mathrm{DS}}}\right)\right\} .
\end{aligned}
$$


At breakdown, the slope of the drain current approaches infinity: $\left(d I_{D} / d V_{\mathrm{DS}}\right) \rightarrow \infty$. In addition, at breakdown, the drain current also approaches infinity: $I_{D} \rightarrow \infty$. From these two conditions and (13) and (14), one obtains $f_{0}=0$, and hence the following equation as the condition for breakdown:

$$
K_{1} K_{2}(M-1) \beta_{f}+\left(1-K_{1}\right)\left(M_{B}-1\right) \beta_{f}=1
$$

From this equation, with the multiplication factors of impact ionization in the surface MOS channel and the parasitic BJT, the breakdown voltage could be found.

1) Strong Inversion Regime: When the device is biased in the strong inversion region $\left(V_{G}>V_{T}\right)$, since multiplication factors $\left(M, M_{B}\right)$ of impact ionization in the surface MOS channel and the parasitic BJT are transcendent functions of the drain voltage ( $V_{\mathrm{DS}}$ ), a solution of (15) in terms of $V_{\mathrm{DS}}$ is difficult to get. When the surface MOS channel dominates, the second term is negligible. Then (15) is simplified as

$\beta_{f} K_{1} K_{2} \frac{\alpha}{\beta}\left(B V_{\mathrm{DSm}}-V_{\mathrm{DSAT}}\right) \exp \left[-\frac{\beta l}{\left(B V_{\mathrm{DSm}}-V_{\mathrm{DSAT}}\right)}\right]=1$.

The exponential term in (16) can be approximated using Taylor's expansion as

$$
\begin{aligned}
\exp \left[-\frac{\beta l}{\left(B V_{\mathrm{DSm}}-V_{\mathrm{DSAT}}\right)}\right] \cong & \exp \left[-\frac{\beta l}{B V_{\mathrm{DSm}}}\right] \\
& \times \exp \left[-\frac{\beta l V_{\mathrm{DSAT}}}{B V_{\mathrm{DSm}}^{2}}\right] .
\end{aligned}
$$

Applying log function to both sides of (16), and using the approximation: $\ln (x)=2((x-1) /(x+1))$ and $\ln (1-x)=$ $-x-\left(x^{2} / 2\right),(16)$ becomes

$$
\begin{aligned}
(G-2) & B V_{\mathrm{DSm}}^{3}+\left(G+2+V_{\mathrm{DSAT}}+\beta l\right) B V_{\mathrm{DSm}}^{2} \\
& +\left(V_{\mathrm{DSAT}}+\frac{1}{2} V_{\mathrm{DSAT}}^{2}+\beta l+\beta l V_{\mathrm{DSAT}}\right) B V_{\mathrm{DSm}} \\
& +\left(\frac{1}{2} V_{\mathrm{DSAT}}^{2}+\beta l V_{\mathrm{DSAT}}\right)=0
\end{aligned}
$$

where $G=\ln \left(1 /\left(K_{1} K_{2}\right) \beta / \alpha\left(\left(1 / \alpha_{f}\right)-1\right)\right)$. From (18), the breakdown voltage for the surface MOS channel-dominated regime is

$$
\begin{aligned}
B V_{\mathrm{DSm}} & =\left(-\frac{m}{2}+\sqrt{D}\right)^{1 / 3}+\left(-\frac{m}{2}-\sqrt{D}\right)^{1 / 3} \\
D & =\left(\frac{n}{3}\right)^{3}+\left(\frac{m}{2}\right)^{2} \\
n & =\frac{1}{3}\left[3\left(\frac{S_{3}}{S_{1}}\right)-\left(\frac{S_{2}}{S_{1}}\right)^{2}\right] \\
m & =\frac{1}{27}\left[2\left(\frac{S_{2}}{S_{1}}\right)^{3}-9\left(\frac{S_{2}}{S_{1}}\right)\left(\frac{S_{3}}{S_{1}}\right)+27\left(\frac{S_{4}}{S_{1}}\right)\right] \\
S_{1} & =G-2 \\
S_{2} & =G+2+V_{\mathrm{DSAT}}+\beta l \\
S_{3} & =V_{\mathrm{DSAT}}+\frac{1}{2} V_{\mathrm{DSAT}}^{2}+\beta l+\beta l V_{\mathrm{DSAT}} \\
S_{4} & =\frac{1}{2} V_{\mathrm{DSAT}}^{2}+\beta l V_{\mathrm{DSAT}} .
\end{aligned}
$$

When the parasitic BJT dominates, (15) becomes

$$
\begin{aligned}
& \frac{1}{\alpha_{f}}-1=\left(1-K_{1}\right) \frac{\alpha_{b}}{\beta_{b}}\left.B V_{\mathrm{DSmb}}-V_{\mathrm{BE}}\right) \\
& \times \exp \left[-\frac{\beta_{b} l_{b}}{\left(B V_{\mathrm{DSmb}}-V_{\mathrm{BE}}\right)}\right] .
\end{aligned}
$$

Using a similar approach as for the case dominated by the surface MOS channel, one obtains a polynomial equation in terms of the breakdown voltage as

$$
\begin{aligned}
\left(G_{b}-2\right) & B V_{\mathrm{DSmb}}^{3}+\left(G_{b}+2+V_{\mathrm{BE}}+\beta_{b} l_{b}\right) B V_{\mathrm{DSmb}}^{2} \\
& +\left(V_{\mathrm{BE}}+\frac{1}{2} V_{\mathrm{BE}}^{2}+\beta_{b} l_{b}+\beta_{b} l_{b} V_{\mathrm{BE}}\right) B V_{\mathrm{DSmb}} \\
& +\left(\frac{1}{2} V_{\mathrm{BE}}^{2}+\beta_{b} l_{b} V_{\mathrm{BE}}\right)=0
\end{aligned}
$$

where $G_{b}=\ln \left(1 /\left(1-K_{1}\right)\left(\beta_{b} / \alpha_{b}\right)\left(\left(1 / \alpha_{f}\right)-1\right)\right)$. From (21), the breakdown voltage for the parasitic-BJT-dominated regime is

$$
\begin{aligned}
B V_{\mathrm{DSmb}} & =\left(-\frac{m_{b}}{2}+\sqrt{D_{b}}\right)^{1 / 3}+\left(-\frac{m_{b}}{2}-\sqrt{D_{b}}\right)^{1 / 3} \\
D_{b} & =\left(\frac{n_{b}}{3}\right)^{3}+\left(\frac{m_{b}}{2}\right)^{2} \\
n_{b} & =\frac{1}{3}\left[3\left(\frac{S_{3 b}}{S_{1 b}}\right)-\left(\frac{S_{2 b}}{S_{1 b}}\right)^{2}\right] \\
m_{b} & =\frac{1}{27}\left[2\left(\frac{S_{2 b}}{S_{1 b}}\right)^{3}-9\left(\frac{S_{2 b}}{S_{1 b}}\right)\left(\frac{S_{3 b}}{S_{1 b}}\right)+27\left(\frac{S_{4 b}}{S_{1 b}}\right)\right] \\
S_{1 b} & =G_{b}-2 \\
S_{2 b} & =G_{b}+2+V_{\mathrm{BE}}+\beta_{b} l_{b} \\
S_{3 b} & =V_{\mathrm{BE}}+\frac{1}{2} V_{\mathrm{BE}}^{2}+\beta_{b} l_{b}+\beta_{b} l_{b} V_{\mathrm{BE}} \\
S_{4 b} & =\frac{1}{2} V_{\mathrm{BE}}^{2}+\beta_{b} l_{b} V_{\mathrm{BE}} .
\end{aligned}
$$

From the surface MOS channel-dominated and the parasitic BJT-dominated breakdown voltages (19) and (22), the universal breakdown voltage formula is

$$
B V_{\mathrm{DS}}=B V_{\mathrm{DSm}}+B V_{\mathrm{DSmb}}-\left(B V_{\mathrm{DSm}}^{6}+B V_{\mathrm{DSmb}}^{6}\right)^{1 / 6}
$$

2) Subthreshold Regime: The breakdown formula derived above is for the device biased in the strong inversion regime. When the device is biased in the weak inversion regime, (19) and (22) are not applicable. At weak inversion ( $V_{\mathrm{DSAT}}$ negligible), if the surface MOS channel dominates, using a similar approach as for the strong inversion regime, (16) is simplified and the breakdown voltage of the surface MOS channel-dominated regime is

$B V_{\mathrm{DSm}}=$
$\quad \frac{-(G+2+\beta l)+\sqrt{(G+2+\beta l)^{2}-4(G-2) \beta l}}{2(G-2)}$. 
TABLE I

PARAMETERS USED IN THE BREAKDOWN MODEL OF THE PD SOI NMOS DEVICE

\begin{tabular}{ccc}
\hline & & \\
Symbol & Value & Unit \\
\hline$K_{1}$ & 0.2 & \\
$K_{2}$ & 0.8 & \\
$\mu_{0}$ & 300 & $\mathrm{~cm}^{2} / V$ sec \\
$\theta$ & 0.1 & $1 / V$ \\
$\theta_{B}$ & 0.1 & $1 / V$ \\
$\phi_{a}$ & 0.2 & \\
$\tau_{0}$ & 1 & $\mu s e c$ \\
$\tau_{g}$ & 10 & $n s e c$ \\
$\tau_{r}$ & 100 & $n s e c$ \\
$\alpha$ & $2.5 \times 10^{6}$ & $\mathrm{~cm}$ \\
$\beta$ & $3.5 \times 10^{6}$ & $V / \mathrm{cm}$ \\
$l$ & $1 \times 10^{-6}$ & $\mathrm{~cm}$ \\
$\alpha_{b}$ & $3 \times 10^{5}$ & $\mathrm{~cm}$ \\
$\beta_{b}$ & $5 \times 10^{5}$ & $V / \mathrm{cm}$ \\
$l_{b}$ & $5 \times 10^{-6}$ & $\mathrm{~cm}$ \\
$E_{C}$ & $1 \times 10^{6}$ & $V / \mathrm{cm}$ \\
\hline
\end{tabular}

At weak inversion, if the parasitic BJT dominates, $V_{\mathrm{BE}}$ is negligible, thus (20) is simplified and the breakdown voltage for the parasitic BJT-dominated regime is

$$
\begin{aligned}
& B V_{\mathrm{DSmb}}= \\
& \frac{-\left(G_{b}+2+\beta_{b} l_{b}\right)+\sqrt{\left(G_{b}+2+\beta_{b} l_{b}\right)^{2}-4\left(G_{b}-2\right) \beta_{b} l_{b}}}{2\left(G_{b}-2\right)} .
\end{aligned}
$$

Thus, (23)-(25) are the breakdown voltage formulas for the device biased in the weak inversion regime.

\section{MODEL EvaluATION}

In order to evaluate the effectiveness of the analytical breakdown model developed here, the model results have been compared with the 2-D simulation results and experimentally measured data. The test device under study is a PD SOI NMOS device with a front gate oxide of $120 \AA$, a thin film of $1200 \AA$ doped with a p-type density of $10^{17} \mathrm{~cm}^{-3}$, and a channel length of 0.7 $\mu \mathrm{m}$. The parameters used in the analytical model are listed in Table I, where fitting parameters are determined from [13] and 2-D simulation results. Fig. 3 shows (a) the drain current and (b) the related $V_{\mathrm{BE}}$ of the parasitic BJT characteristics of this test device based on the compact model and the MEDICI 2-D results. As shown in this figure, the breakdown of the device is strongly correlated to $V_{\mathrm{BE}}$ of the parasitic BJT, which is also related to the kink effect [11], [12]. At the larger $V_{\mathrm{GS}}$ of $3 \mathrm{~V}$, the related $V_{\mathrm{BE}}$ is smaller, which indicates a weak function of the parasitic BJT while the surface MOS channel is dominant. At the smaller $V_{\mathrm{GS}}$ of $0.5 \mathrm{~V}$, the parasitic BJT turns on earlier and the contribution of impact ionization from the parasitic BJT becomes more important. As shown in the figure, at a high drain voltage, the deviation in the base-emitter voltage between the

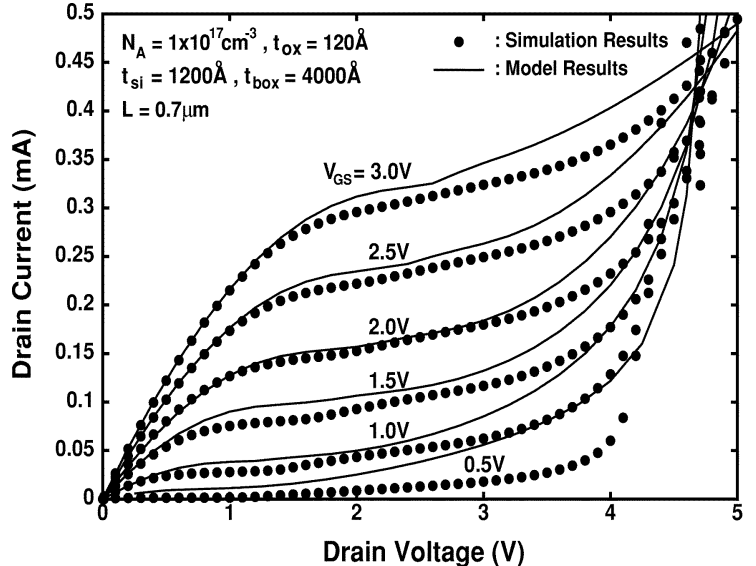

(a)

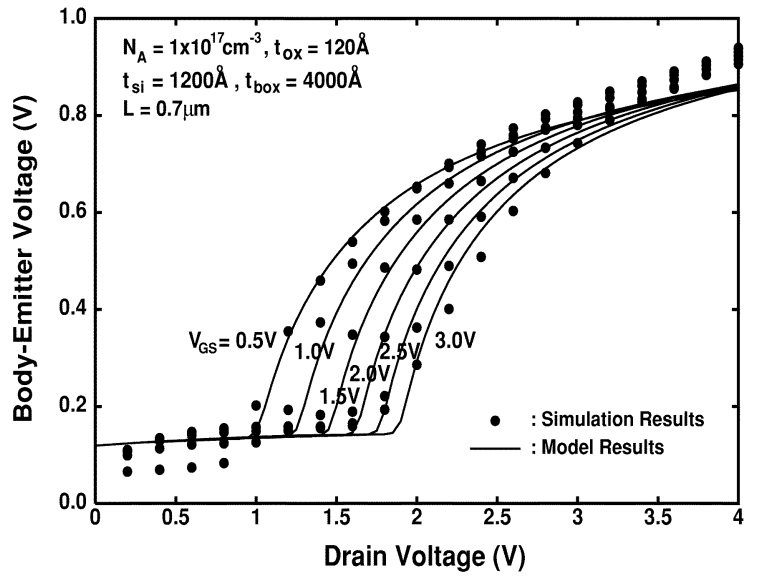

(b)

Fig. 3. (a) Drain current and (b) related body-emitter voltage versus drain voltage of the PD SOI NMOS device with a front gate oxide of $120 \AA$, a thin film of $1200 \AA$ doped with a p-type density of $10^{17} \mathrm{~cm}^{-3}$, and a channel length of $0.7 \mu \mathrm{m}$ based on the analytical model and 2-D simulation results.

model and the simulation results becomes noticeable, which results in a substantial deviation in the threshold voltage. The deviation is due to the over-simplified parasitic bipolar device modeled used for describing the complicated floating-body effect mechanism. In addition, drain-induced barrier lowering (DIBL) is not considered. Nevertheless, the analytical model still could predict the breakdown voltage with a reasonable accuracy.

Fig. 4 shows the breakdown voltage versus the gate voltage of the PD SOI NMOS device as described for Fig. 3 with channel lengths of $0.7 \mu \mathrm{m}$ and $2.7 \mu \mathrm{m}$, based on the compact model, the 2-D simulation results, and the experimentally measured data [9]. As shown in Fig. 4, in the strong inversion, when the gate voltage decreases, the breakdown voltage decreases due to a larger influence from the parasitic BJT. In the subthreshold region, when the gate voltage becomes smaller, the breakdown voltage increases due to a weaker function of the parasitic BJT. As shown in this figure, the analytical model predicts well the breakdown voltage as verified by the 2-D simulation results and the experimentally measured data.

\section{DISCUSSION}

Fig. 5 shows the breakdown voltage versus the channel length of the PD SOI NMOS devices (a) with various thin film doping 


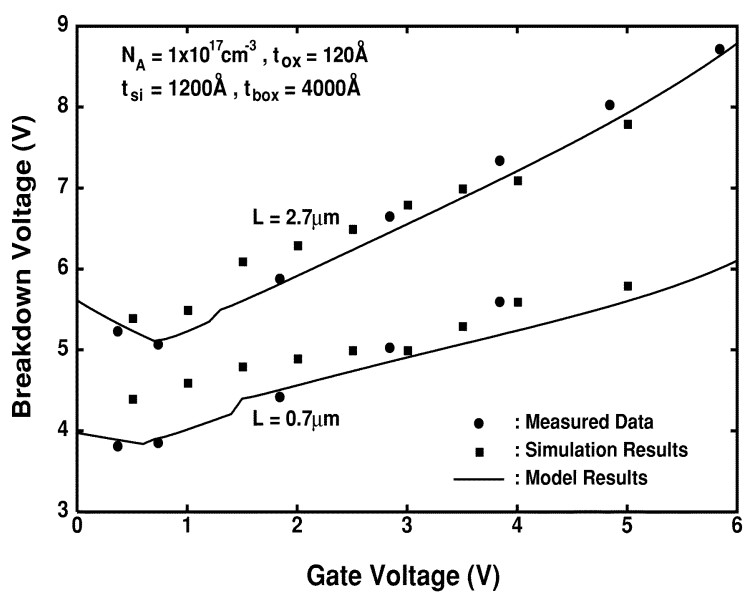

Fig. 4. Breakdown voltage versus gate voltage of the PD SOI NMOS device with a gate oxide of $120 \AA$, a thin film of $1200 \AA$ doped with a p-type density of $10^{17} \mathrm{~cm}^{-3}$, and channel lengths of $0.7 \mu \mathrm{m}$ and $2.7 \mu \mathrm{m}$, based on the analytical model, the MEDICI 2-D simulation results, and the experimentally measured data.

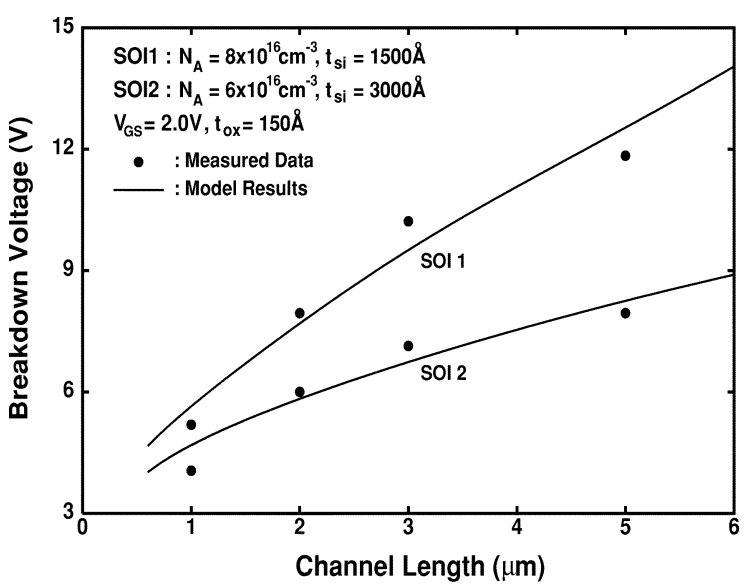

(a)

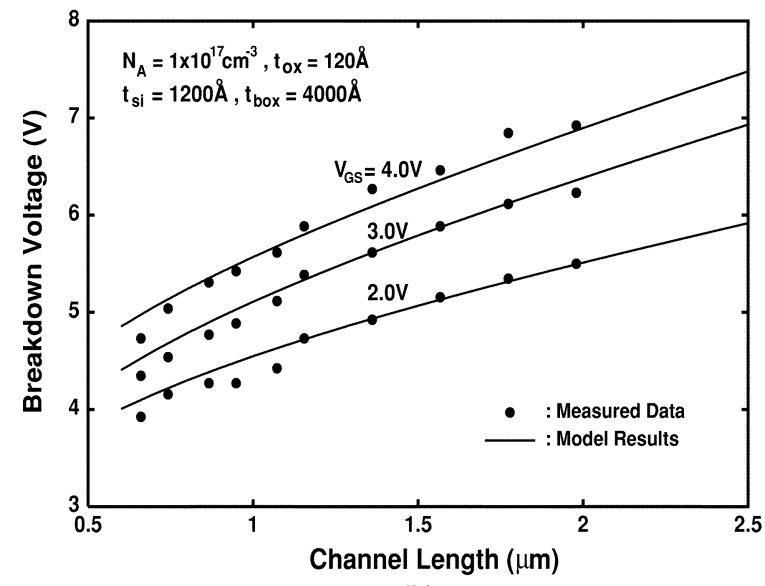

(b)

Fig. 5. Breakdown voltage versus channel length of the PD SOI NMOS device: (a) with various thin film doping densities and thin film thicknesses, biased at $V_{\mathrm{GS}}=2 \mathrm{~V}$ and (b) with various $V_{\mathrm{GS}}^{\prime} \mathrm{s}$, based on the compact model and the experimentally measured data.

densities and thin film thicknesses biased at $V_{\mathrm{GS}}=2 \mathrm{~V}$ and (b) with various $V_{\mathrm{GS}}^{\prime} \mathrm{s}$, based on the compact model and the experimentally measured data [3], [9]. As shown in Fig. 5(a), when the channel length is larger, the breakdown voltage increases.

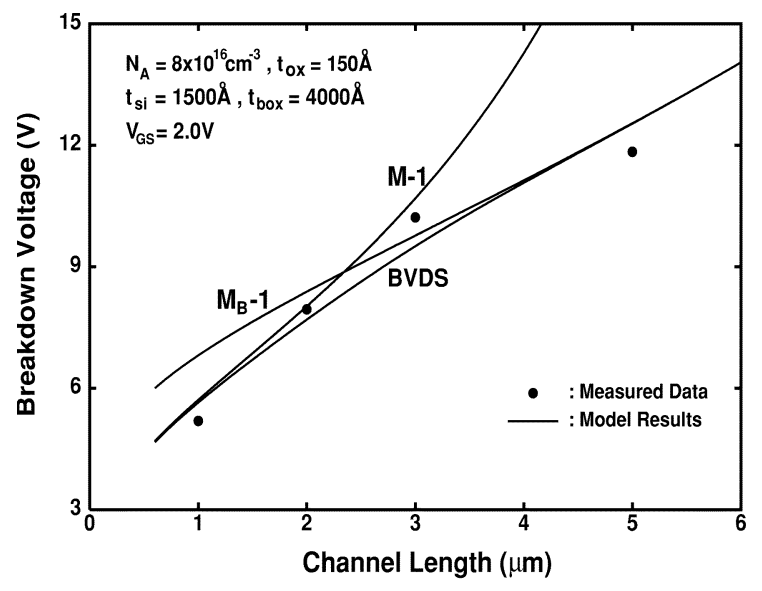

Fig. 6. Breakdown voltage by considering both the surface MOS channel and the parasitic BJT (BVDS), by considering only the surface MOS channel $(M-$ $1)$, and by considering only the parasitic BJT $\left(M_{B}-1\right)$ versus channel length of the PD SOI NMOS device with a gate oxide of $150 \AA$, a thin film of $1500 \AA$ doped with a p-type density of $8 \times 10^{16} \mathrm{~cm}^{-3}$, based on the analytical model and the experimental data.

With a thicker thin film, the breakdown voltage is smaller due to a stronger parasitic BJT. As shown in Fig. 5(b), at a smaller $V_{\mathrm{GS}}$, the breakdown voltage becomes smaller since the function of the parasitic BJT becomes stronger.

Fig. 6 shows the breakdown voltage by considering both the surface MOS channel and the parasitic BJT (BVDS), by considering only the surface MOS channel $(M-1)$, and by considering only the parasitic $\mathrm{BJT}\left(M_{B}-1\right)$ versus the channel length of the PD SOI NMOS device with a gate oxide of $150 \AA$, and a thin film of $1500 \AA$ doped with a p-type density of $8 \times 10^{16} \mathrm{~cm}^{-3}$ based on the analytical model and the experimental data [3]. As shown in Fig. 6, when the channel length is small, the breakdown voltage of the device is predominantly determined by the surface MOS channel since the impact ionization at the surface is more important due to a large lateral electric field. When the channel length becomes large, dominance of impact ionization by the surface MOS channel is replaced by that of the parasitic BJT.

The analytical breakdown model is not just for a long channel case. It is applicable for a short channel case as well. Fig. 7 shows the drain current versus the drain voltage of a $0.25-\mu \mathrm{m}$ PD SOI NMOS device with a front gate oxide of $50 \AA$, and a thin film of $800 \AA$, doped with a p-type density of $5 \times 10^{17} \mathrm{~cm}^{-3}$, based on the analytical model and the 2-D simulation results. As shown in Fig. 7, for a channel length of $0.25 \mu \mathrm{m}$, the analytical model still could predict the breakdown behavior well.

Fig. 8 shows the ratio of the breakdown voltage determined by only the surface MOS channel to that by only the parasitic $\mathrm{BJT}\left(B V_{\mathrm{DSm}} / B V_{\mathrm{DSmb}}\right)$ versus the channel length of the PD SOI NMOS device with various thin film structures based on the compact model. As shown in this figure, when the channel length is small, $\left(B V_{\mathrm{DSm}} / B V_{\mathrm{DSmb}}\right)$ is less than one, which implies dominance of the surface MOS channel. The solid lines show the cases with the multiplication factor coefficient of the surface MOS channel equal to 1.5 times that of the parasitic BJT $\left((\alpha / \beta)_{M} /(\alpha / \beta)_{M_{B}}=1.5\right)$. Under this situation, when the thickness of the thin film is increased from $1500 \AA$ to $3000 \AA$, the $B V_{\mathrm{DSm}} / B V_{\mathrm{DSmb}}$ curve moves upward from SOI 1 


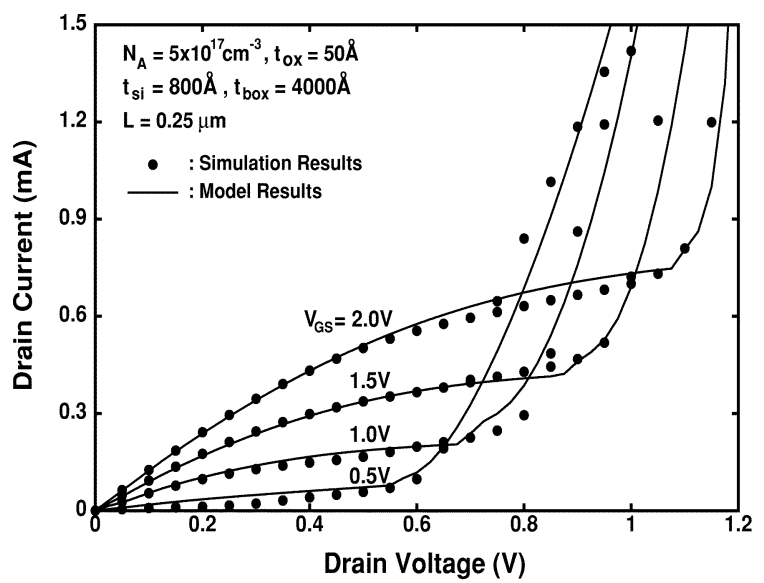

Fig. 7. Drain current versus drain voltage of the PD SOI NMOS device with a front gate oxide of $50 \AA$, a thin film of $800 \AA$, doped with a p-type density of $5 \times 10^{17} \mathrm{~cm}^{-3}$, and channel length of $0.25 \mu \mathrm{m}$ based on the analytical model and 2-D simulation results.

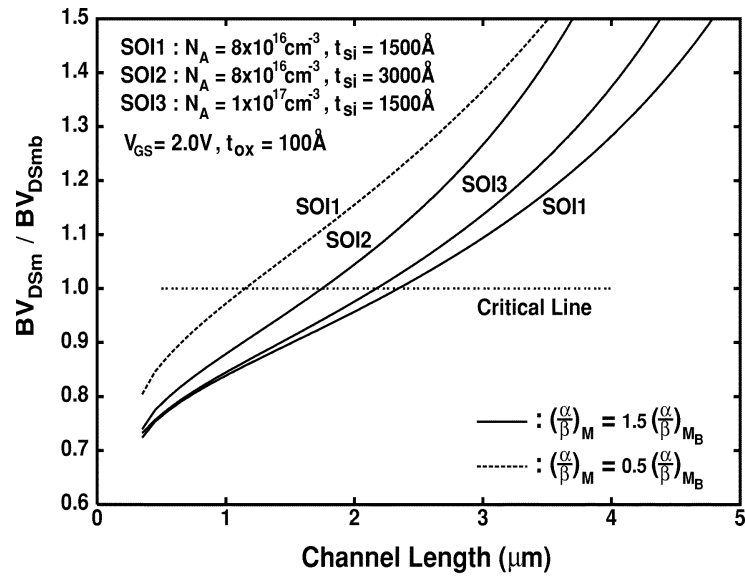

Fig. 8. Ratio of the breakdown voltage determined by only the surface MOS channel to that by only the parasitic BJT versus the channel length of the PD SOI NMOS device with various thin film structures based on the analytical model.

to SOI2, which implies the relative weight of parasitic BJT tends to become more important. When the thin film doping density is increased from $8 \times 10^{16} \mathrm{~cm}^{-3}$ to $10^{17} \mathrm{~cm}^{-3}$, the $B V_{\mathrm{DSm}} / B V_{\mathrm{DSmb}}$ curve also moves upward from SOI1 to $\mathrm{SOI} 3$, which can be reasoned as follows. With a higher thin ilm doping density, although the function of the parasitic BJT is reduced, the extent of the increase in the threshold voltage of the surface MOS device is even more. As a result, with an increase in the thin film doping density, the relative weight of the parasitic BJT in terms of impact ionization increases when the thin film doping density is raised. When the multiplication factor coefficient of the surface MOS channel is reduced to 0.5 times that of the parasitic BJT $\left((\alpha / \beta)_{M} /(\alpha / \beta)_{M_{B}}=0.5\right)$, the $B V_{\mathrm{DSm}} / B V_{\mathrm{DSmb}}$ curve (dashed line) is higher than the original one-SOI1 (solid line) with $(\alpha / \beta)_{M} /(\alpha / \beta)_{M_{B}}=1.5 \mathrm{~V}$.

\section{CONCLUSION}

In this paper, a compact breakdown voltage model for PD SOI NMOS devices considering BJT/MOS impact ionization has been presented. Via the improved current conduction model considering BJT/MOS impact ionization, this compact model provides an accurate prediction of the breakdown behavior of the PD SOI NMOS devices as verified by the experimental data and the MEDICI results. Based on the analytical model, in the strong inversion region, when the gate voltage is lowered, the breakdown voltage decreases. In the subthreshold region, a smaller gate voltage leads to a larger breakdown voltage due to a weaker function of the parasitic BJT.

\section{REFERENCES}

[1] J. B. Kuo and S. C. Lin, Low-Voltage SOI CMOS VLSI Devices and Circuits. New York: Wiley, 2001.

[2] J. B. Kuo and K. W. Su, CMOS VLSI Engineering: Silicon-on-Insulator (SOI). Norwell, MA: Kluwer, 1998.

[3] K. K. Young and J. A. Burns, "Avalanche-induced drain-source breakdown in silicon-on-insulator n-MOSFETs," IEEE Trans. Electron Devices, vol. 35, pp. 426-431, Apr. 1988.

[4] J. S. T. Huang, H. J. Chen, and J. S. Kueng, "Modeling of output snapback characteristics in n-channel SOI MOSFETs," IEEE Trans. Electron Devices, vol. 39, pp. 1170-1178, May 1992.

[5] S. Maeda, Y. Hirano, Y. Yamaguchi, T. Iwamatsu, T. Ipposhi, K. Ueda, K. Mashiko, S. Maegawa, H. Abe, and T. Nishimura, "Substrate-bias effect and source-drain breakdown characteristics in body-tied shortchannel SOI MOSFETs," IEEE Trans. Electron Devices, vol. 46, pp. 151-158, Jan. 1999.

[6] K. Mistry, G. Grula, J. Sleight, L. Barr, R. Stephany, R. Flatley, and P. Skerry, "A $2.0 \mathrm{~V}, 0.35 \mu \mathrm{m}$, partially depleted SOI CMOS technology," in IEDM Tech. Dig., 1997, pp. 583-586.

[7] W. Chen, Y. Taur, D. Sadana, K. A. Jenkins, J. Sun, and S. Cohen, "Suppression of the SOI floating-body effects by linked-body device structure," in Proc. VLSI Technol. Symp., 1996, pp. 92-93.

[8] M. Terauchi, M. Yoshimi, A. Murakoshi, and Y. Ushiku, "Suppression of the floating-body effects in SOI MOSFETs by band gap engineering," in Proc. VLSI Technol. Symp., 1995, pp. 35-36.

[9] J. Chen, F. Assaderaghi, H. -J Wann, P. Ko, and C. Hu, "An accurate model of thin film SOI MOSFET breakdown voltage," in IEDM Tech. Dig., 1991, pp. 671-674.

[10] H.-K. Yu, J.-S. Lyu, S.-W. Kang, and C.-K. Kim, "A physical model of floating-body thin film silicon-on-insulator nMOSFET with parasitic bipolar transistor," IEEE Trans. Electron Devices, vol. 41, pp. 726-733, May 1994.

[11] S. S. Chen and J. B. Kuo, "An analytical CAD kink effect model of partially depleted SOI NMOS devices operating in strong inversion," Solid State Electron., vol. 41, pp. 447-458, Mar. 1997.

[12] S. S. Chen, S. C. Lin, and J. B. Kuo, "Kink effect on subthreshold current conduction mechanism for n-channel metal-oxide-silicon devices," $J$. Appl. Phys., vol. 80, pp. 5821-5827, Nov. 1996.

[13] Y. P. Tsividis, Operation and Modeling of the MOS Transistor. New York: McGraw-Hill, 1988.

[14] A. S. Grove, Physics and Technology of Semiconductor Devices. New York: Wiley, 1967.

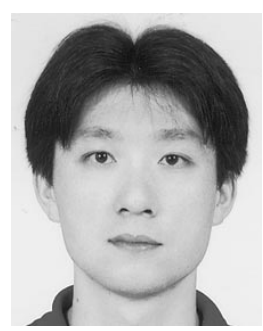

Shih-Chia Lin received the B.S.E.E. and Ph.D.E.E. degrees from National Taiwan University, Taipei, Taiwan, R.O.C., in 1992 and 2001, respectively.

In 2001, he joined the University of Waterloo, Waterloo, ON, Canada, as a Research Assistant Professor. His research expertise is in compact modeling of CMOS VLSI devices. 


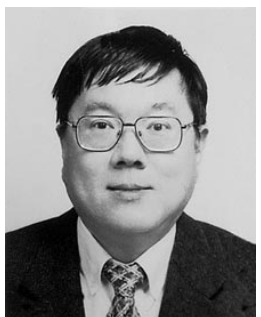

James B. Kuo (S'85-M'85-SM'92-F'00) received the B.S.E.E. degree from National Taiwan University, Taipei, Taiwan, R.O.C., in 1977, the M.S.E.E. degree from The Ohio State University, Columbus, in 1978 and the Ph.D.E.E. degree from Stanford University, Stanford, CA, in 1985

In 1987, he joined National Taiwan University as an Associate Professor, and later became a Professor (in 1990). In 2000, he joined the University of Waterloo, Waterloo, ON, Canada, as a tenured Full Professor, on leave from National Taiwan University. He has published 250 technical papers including 50 IEEE journal papers. He holds 16 invention patents including seven U.S. patents on low-voltage CMOS VLSI circuits. As a highly recognized expert, he has authored nine books, including Low-Voltage SOI CMOS VLSI Devices and Circuits (New York: Wiley, 2001), Low-Voltage CMOS VLSI Circuits (New York: Wiley, 1999), and CMOS VLSI Engineering: Silicon-On-lnsulator (SOI) (Norwell, MA: Kluwer, 1998). As a technical leader, he has graduated 50 M.S. and Ph.D. students specialized in CMOS circuit designs and device modeling, currently working in leading U.S and Taiwan microelectronics companies. In 2001, he was awarded the prestigious Canada Research Chair Professor by the Canadian government. His research expertise is in the field of low-voltage CMOS VLSI circuits and SPICE compact modeling of deep-submicrometer bulk and SOI CMOS and BiCMOS VLSI devices. As a leader of his research group at National Taiwan University, he has accomplished several breakthroughs in low-voltage CMOS digital circuits.

Dr. Kuo is an IEEE Fellow for contributions to modeling CMOS VLSI devices. He is also an IEEE distinguished lecturer. He serves as an Associate Editor for the IEEE Circuits and Devices Magazine, and the membership committee chair for the IEEE Electron Devices Society. 